\title{
Centerline extraction based three-dimensional architecture parameter measurement method for plant roots
}

\author{
Zishang Yang ${ }^{1}$, Xuecheng Zhou ${ }^{2 *}$, Fuqiang Chen ${ }^{2}$, Yuxing $\operatorname{Han}^{1 *}$ \\ (1. College of Electronic Engineering, South China Agricultural University, Guangzhou 510642, China; \\ 2. College of Engineering, South China Agricultural University, Guangzhou 510642a, China)
}

\begin{abstract}
The detection of architecture was one of the essential questions of plant root phenotyping research. The classical root architecture detection method was carried out by manual measurement. It is not only tedious, but also has a poor reliability, and the roots are damaged easily. This paper described a three-dimensional architecture measurement method based on XCT and centerline extraction method. The method includes the following steps: (1) obtaining the root CT images through the XCT system; (2) obtaining a root three-dimensional model after image segmentation and reconstruction. To solve the problem of model fracture, the quality of the reconstruction model was improved by a series of pre-processing methods; (3) extracting the root's centerline based on the mesh contraction, and the high-quality centerline was obtained after the post-processing methods; (4) calculating the architecture parameters. Different root samples were tested to validate the method for centerline extraction, and the root architecture was calculated by the centerline. The results were compared with the manual measurements, and the mean absolute percentage error of root length and root angle were $1.74 \%$ and 4.51 , respectively. The entire algorithm runs for less than 30 seconds. The study may provide an effective method for root architecture detection.
\end{abstract}

Keywords: CT Images, three-dimensional root model, centerline extraction, root architecture, plant root phenotyping DOI: $10.33440 /$ j.ijpaa.20190202.38.

Citation: Yang Z S, Zhou X C, Chen F Q, Han Y X. Centerline extraction based three-dimensional architecture parameter measurement method for plant roots. Int J Precis Agric Aviat, 2019; 2(2): 11-18.

\section{Introduction}

The root system is an important organ for plant growth, and it has essential functions such as absorption and transport of water, nutrients and storage of organic compounds ${ }^{[1]}$. Plant root has different growth architectures, which has huge differences in nutrient, water and phosphorus absorption efficiency ${ }^{[2-3]}$. There are two important issues of root system architecture research, the structure and the traits of the root system ${ }^{[4]}$. The former refers to the various components of the root system and the relationship between them. The latter relates to the spatial modeling and distribution of the root system in the growth medium, including two-dimensional (2D) and three-dimensional (3D) architecture. Common root three-dimensional architecture parameters include the number of root branches, root branch length, root branch angle, root branch surface area, and volume ${ }^{[5]}$, etc.

To accurately obtain the architecture parameters of plant roots grown in soil media, many scholars have proposed varieties of detection methods in the early year ${ }^{[5]}$, such as the excavation method, the auger or soil-core sampling method, the glass wall method, the underground root laboratories, and the minirhizotrons method. These methods are time consuming and laborious, and the measurement accuracy is easily affected by manual operations and had been widely used up to now.

Received date: 2019-11-25 Accept date: 2019-12-15

Biographies: Zishang Yang, Doctoral student, research interests: Computer Vision, Email: yzs@stu.scau.edu.cn; Fuqiang Chen, research interests: Computer Vision, Email: 775848471@qq.com.

*Corresponding authors: Yuxing Han, PhD, Professor, research interests: Computer Vision, Email: yuxinghan@scau.edu.cn; Xuecheng Zhou, PhD, Professor, research interests: Computer Vision, Email: zxcem@scau.edu.cn.
With the development of 2D imaging technology, researchers could acquire images easily through $\mathrm{CCD}$ cameras or flat scanners ${ }^{[6]}$. After that, the region of interest (ROI) was preserved after image segmentation, and $2 \mathrm{D}$ root architecture parameters were measured. There are a series of 2D measurement platforms for plant roots including RootRrace ${ }^{[7]}$, EZ-Rhizo ${ }^{[8]}$, Smart Root ${ }^{[9]}$, IJ_Rhizo ${ }^{[10]}$, and DIRT ${ }^{[11]}$, etc. Wasson et al. developed a portable fluorescence spectroscopy imaging system for automated root phenotypic analysis ${ }^{[12]}$. However, due to the limitation of $2 \mathrm{D}$ architecture measurement, and its inability to measure 3D architecture parameters, some researchers have explored 3D architecture measurement methods.

For the research in the $3 \mathrm{D}$ architecture of plant roots, some researchers applied laser scanning technology, MRI technology, and X-ray CT technology to the detection of plant roots $\operatorname{architecture}^{[13]}$. Gregory et al. performed non-invasive imaging of roots using high-resolution X-ray tomography ${ }^{[14]}$. Bingham et al. used to simulate wheat growth using the $3 \mathrm{D}$ root architecture model $^{[15]}$. Dunbabin et al. used a three-dimensional model of root growth, structure and function to simulate root-soil interactions ${ }^{[16]}$. $\mathrm{Wu}$ et al. proposed a method to measure the 3D architecture of axile roots of field-grown maize ${ }^{[17]}$. Wu et al. used a $3 \mathrm{D}$ root architecture model to measure the root-length-density distribution of corn ${ }^{[18]}$. Nicolai K proved that X-ray CT technology could be used for plant root research ${ }^{[19]}$, and it was more suitable for micro roots than the MRI ${ }^{[20]}$. In a recent work, Landl et al. proposed a novel method for measuring wheat root traits in $2 \mathrm{D}$ images to parameterize 3D root architecture models ${ }^{[21]}$. To obtain the root $3 \mathrm{D}$ architecture from the original $\mathrm{CT}$ images, the root region is first segmented from the slice images to obtain the ROI, and then the root $3 \mathrm{D}$ model is reconstructed by the images ${ }^{[22]}$. For the root $3 \mathrm{D}$ model, it's often through interactive manual measurements ${ }^{[23]}$, 
which is efficiency low and difficult to guarantee accuracy.

The centerline is a compact representation of the shape of the model, and it is not only reflecting the topological information of the shape, but also is able to describe the geometrical characteristics of the shape. Grélard et al. proposed a centerline extraction method based on the minimum tangent plane for the tubular models ${ }^{[24]}$. Huang et al. proposed a skeleton extraction method for the point cloud models ${ }^{[25]}$, which is constructed by iteratively solving the local $L_{1}$ point. $\mathrm{Au}$ et al. proposed an extraction algorithm based on Laplacian operator ${ }^{[26]}$, which obtained the skeleton of the curve through mesh contraction. Lee et al. applied 2D thinning to 2D objects and proposed a thinning method for constructing the centerline of $3 \mathrm{D}$ objects ${ }^{[27]}$. Although many methods of centerline extraction have been proposed for different research objects and different research purposes, it is still a challenging research field. The skeletons or centerlines extracted by different methods have different performances in many characteristics such as centrality and continuity, and there is still lacking a general algorithm. Therefore, for the basic requirements of the $3 \mathrm{D}$ root architecture measurement of the centrality, continuity and other characteristics of the centerline, it is necessary to explore a method suitable for the 3D root model and then achieve the $3 \mathrm{D}$ architecture parameters extraction of the root system. It has undoubtedly important theoretical and practical significance.

In this study, the root system was taken as the research object. The root $\mathrm{CT}$ images were acquired by the $\mathrm{XCT}$ imaging equipment, and then the 3D surface mesh model of plant roots was reconstructed. According to the root architecture characteristics, a set of quality improvement methods for the centerline of the root system was designed. Automatic measurement and quantitative analysis of roots were implemented. The phenotypic analysis method based on XCT enabled to perform high-throughput and non-destructive measurement of the plant roots, which makes it possible to study the complex theory of plants during the growth process and providing an effective way for root phenotypic detection.

\section{Materials and methods}

\subsection{Equipment and data acquisition}

For the XCT imaging system, it can sense the difference in density between the objects. When X-rays pass through different objects, it can cause different attenuation of the rays. Attenuation of the radiation caused by, for example, air, water, and soil, and the rotation and axial movement of the object relative to the sensor produces $3 \mathrm{D}$ images ${ }^{[28]}$. For the existing medical or industrial XCT devices, it is difficult to maintain a balance between imaging resolution and imaging range and expensive cost. Therefore, an XCT-based root imaging system was used. It includes a ray source, a mechanical scanning system, a detector system, a graphics workstation, and the other. The imaging system adopts the cone beam scanning method. The radiation source adopts the type of Coment MXR-160HP/11, the flat panel detector adopts the type of VARIAN PaxScan $2520 \mathrm{~V}$, and the raw images processing software adopts nVCTiS Studio 6.0. The density resolution of the system arrives $1 \%$ to $5 \%$, and a spatial resolution of its arrives $0.1 \mathrm{~mm}$ to $0.3 \mathrm{~mm}$. The framework of it is shown in Figure 1 .

In this study, different types of plant roots such as Michelia Macclurei seedlings and Ficus Altissima seedlings were selected as experimental samples. A set of roots is shown in Figure 2. It was obtained directly by excavating from the arboretum, and the plants were transplanted into pots with maintaining the original state of the roots, and then placed in the XCT root imaging system to acquire the $\mathrm{CT}$ images.

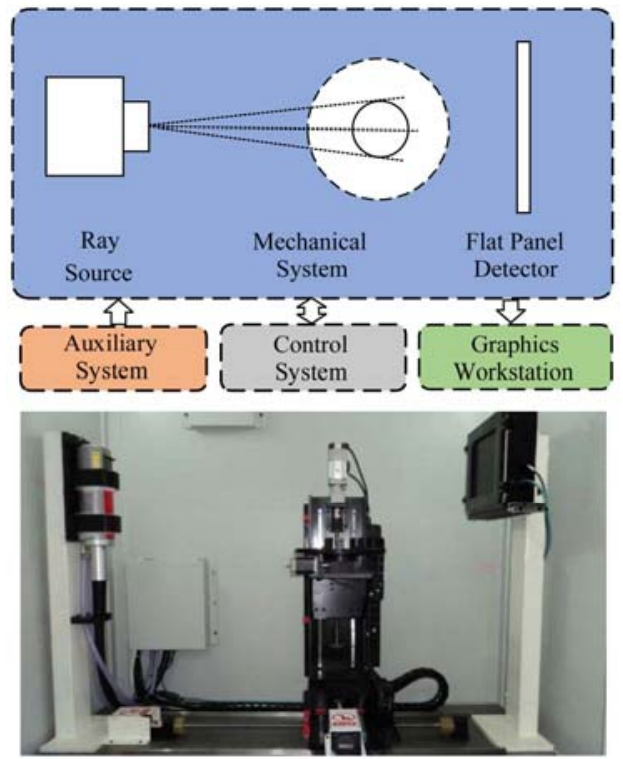

Figure 1 X-ray CT imaging system.
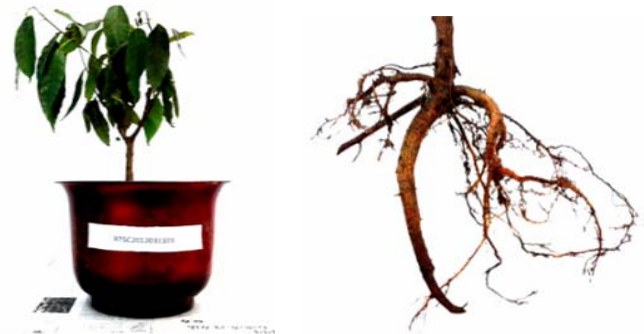

Figure 2 Sample of the plant roots.

\subsection{D architecture information acquisition method for plant roots}

In order to obtain the root architecture from the raw $\mathrm{CT}$ images, the root region is first segmented from the images to obtain the root 3D slice images. The slice images are filtered and morphologically processed in $3 \mathrm{D}$. After that, the root $3 \mathrm{D}$ surface mesh model is reconstructed. An approximate 1D linear mesh model is obtained by multiple iterations of mesh contraction, and a $1 \mathrm{D}$ centerline is obtained after the mesh is removed. A complete smooth centerline is constructed after the post-processing. And then, the important parameters of root architecture such as branch length and branch angle can be calculated. Thereby, the automatic calculation and quantitative analysis of the 3D architecture parameters of plant roots are realized. The processing architecture of the complete method consists of four steps, which are sketched in Figure 3.

\subsubsection{Image segmentation}

A major challenge in X-ray $\mathrm{CT}$ imaging is the processing and analysis of the resulting images ${ }^{[29]}$. At the same time, root CT imaging is different from medical applications, which can distinguish different biological structures easily based on grayscale values $^{[30]}$. Therefore, root CT image segmentation is one of the critical steps in the whole algorithm flow, and the quality of segmentation also affects the extraction of the centerline. For root CT slice images, common methods of segmentation include thresholding methods ${ }^{[31]}$, region growing approaches, watershed segmentation, and some methods based on machine learning ${ }^{[32]}$. Zhou et al. proved that filtering the image before segmentation can 
effectively improve the quality of segmentation ${ }^{[33]}$. Therefore, this study first filters the root CT slice images, and then uses the
$3 \mathrm{D}$ region growing method based on the confidence interval for image segmentation.

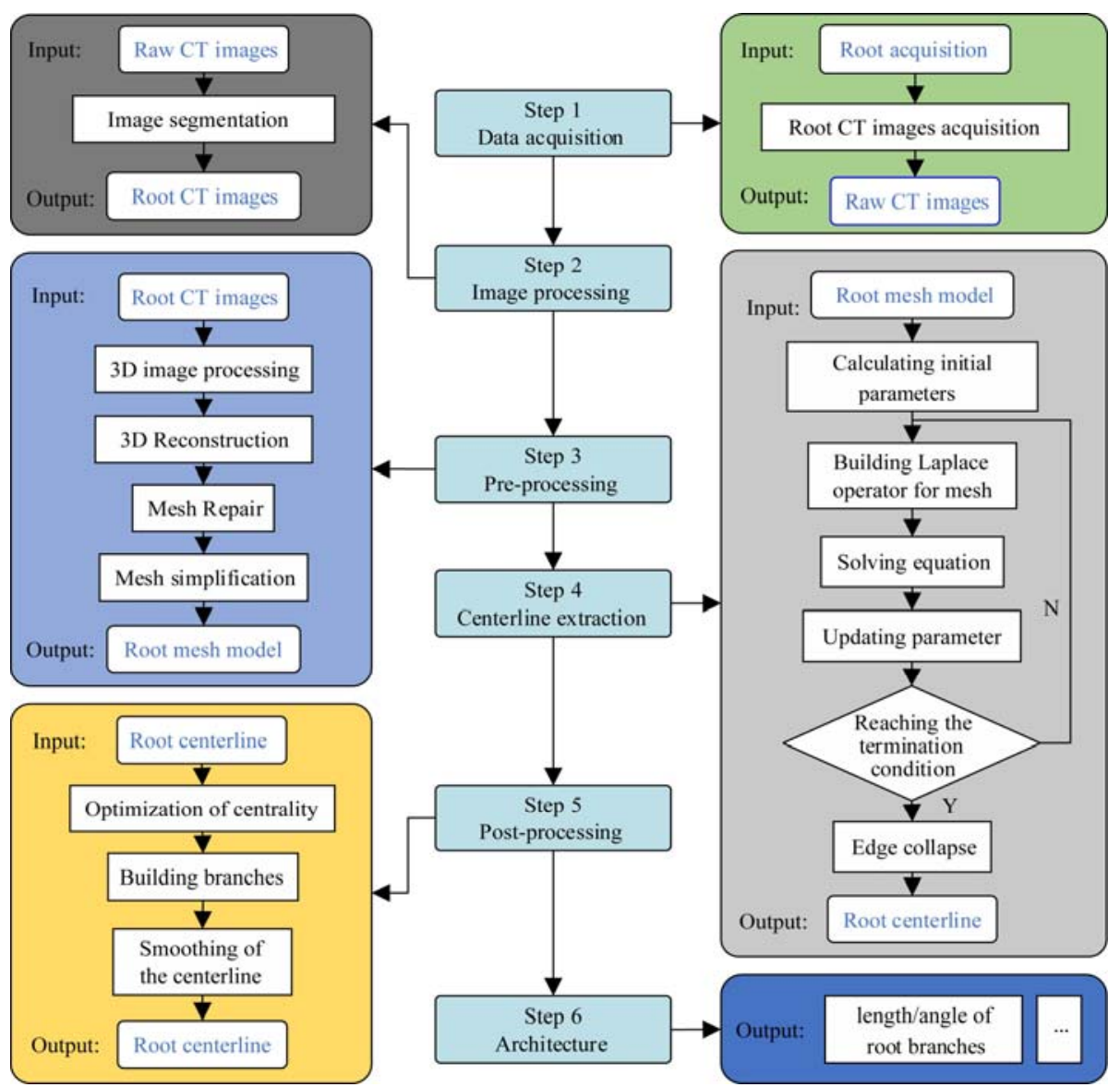

Figure 3 Algorithm flowchart for extracting the 3D architecture information for plant roots

The method takes some statistical concepts on the similarity criterion and requires the user to provide a factor. Its formula is as follows:

$$
I(X) \in[m-f \sigma, m+f \sigma]
$$

where, $m$ and $\sigma$ are the average value and standard deviation value of the area brightness; $f$ is a factor provided by the user, and $I$ is the input image.

Observing and analyzing the CT slice images of different samples, which have the following characteristics:

(1) The root section in the images is the connected area with the largest area;

(2) The evenly growing root system has a columnar linear characteristic, which is reflected in the root section as a region close to a circle;

(3) The main root section of the plant root is generally located closer to the center of the images.

Therefore, according to these characteristics described above, the formula for calculating the factors can be defined as follows:

$$
f=\frac{A}{A_{M a x}} f_{1}+\frac{R}{R_{M a x}} f 2+\frac{L}{L_{M a x}} f_{3}
$$

where, $f$ denotes a factor; the $A, R$ and $L$ respectively represent the area of the currently connected region, the circular ratio, and the distance from the center of the image. The $A_{M a x}, R_{\operatorname{Max}}$, and $L_{M a x}$ represent the respective maximum values in the images, respectively. The $f_{1}, f_{2}$, and $f_{3}$ represent the area influence factor, the round rate influence factor and the distance influence factor. The three impact factor values are set in this paper: $f_{1}=0.5, f_{2}=0.3$, and $f_{3}=0.2$.

In order to verify the segmentation effect, this paper reconstructs the root samples after segmentation to observe the segmentation effect. The result is shown in Figure 4. The region growing method based on the confidence interval can not only effectively remove the impurity regions in the image, but also preserve the complete root region.

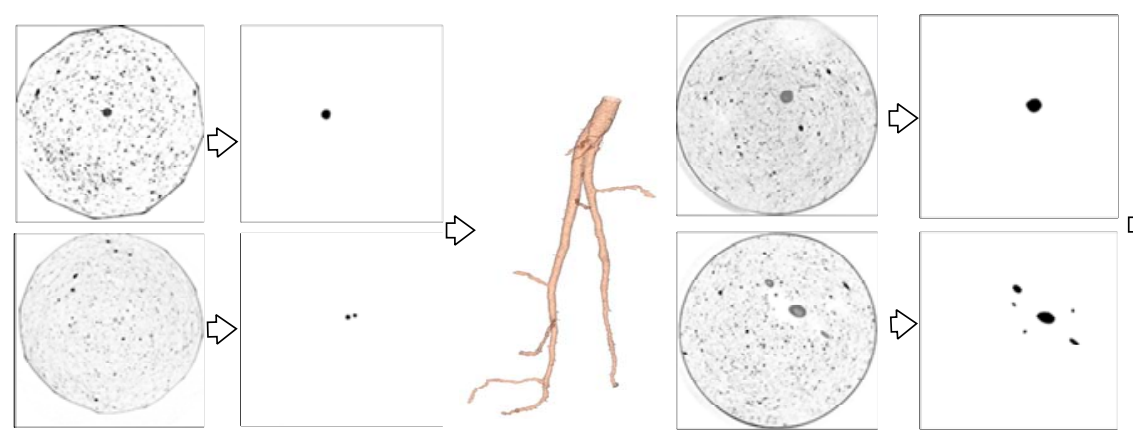

Figure 4 Image segmentation and reconstruction result. 


\subsubsection{D images pre-processing and reconstruction}

There are over-segmentation and under-segmentation problems in the segmented images, which leads to appear the fracture and the noise of the model that obtained by reconstruction. As shown in Figure 5a. After previous tests, these problems are not easily eliminated by $3 \mathrm{D}$ model processing and may cause errors for the centerline extraction. Therefore, this study takes the following pre-processing methods to improve the quality of the reconstructed models.

(1) Performing a 3D spatial median filtering and dilating process on the root $3 \mathrm{D}$ images to reduce noise and fracture problems.

(2) Reconstructing the 3D images by VTK to obtain a surface mesh model ${ }^{[1]}$ and filling the model holes.

(3) Simplify the mesh to reduce redundant data.

Through the pre-processing, the fracture and noise problems in the model are reduced, as shown in Figure 5b. At the same time, the amount of data of the model itself is effectively reduced, as shown in Table 1 for comparison before and after processing.

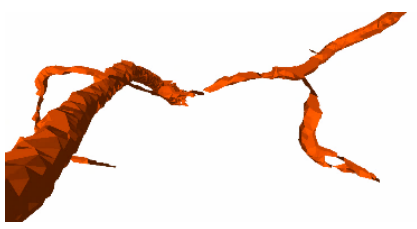

a.

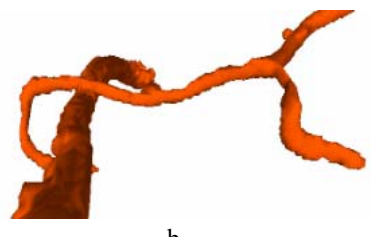

b.
Figure 5 Model pre-processing

Table 1 Comparison of model preprocessing

\begin{tabular}{lcc}
\hline \multicolumn{1}{c}{ Items } & Before & After \\
\hline Number of vertexes & 246234 & 34443 \\
Number of faces & 738876 & 103208 \\
Size of file & $35.8 \mathrm{MB}$ & $2.26 \mathrm{MB}$ \\
Time of loading & $128.23 \mathrm{~s}$ & $10.33 \mathrm{~s}$ \\
\hline
\end{tabular}

2.2.3 Centerline extraction based on mesh contraction

For the root mesh model, the cotangent Laplacian operator is constructed firstly. Then, the contraction equation is solved iteratively, and the mesh model of the approximate $1 \mathrm{D}$ curve is obtained after the iteration is terminated. After that, the unnecessary mesh boundaries are removed by half-edge collapse to obtain a $1 \mathrm{D}$ centerline.

2.2.3.1 Mesh contraction
For a given triangular mesh $U=(V, E, F)$ with n vertices, where $V$ is the set of vertices, $E$ is the set of edges, and $F$ is the set of faces. The cotangent Laplacian matrix $L$ can be expressed as ${ }^{[34]}$ :

$$
L_{i j}= \begin{cases}\omega_{i j}=\cot \alpha_{i j}+\cot \beta_{i j} & (i, j) \in E \\ \sum_{(i, k) \in E}^{k}-\omega_{i j} & i=j \\ 0 & \text { Other }\end{cases}
$$

For the cotangent Laplacian operator, the surface mesh tends to contraction along the normal direction of the curvature ${ }^{[35]}$. Therefore, by solving the Laplace equation $L V^{\prime}=0$, the mesh vertex $V^{\prime}$ is smoothly contraction along the normal direction. Thus, there is the following Laplace mesh contraction equation ( $\mathrm{Au}$ et al. 2008).

$$
\left[\begin{array}{c}
W_{L} L \\
W_{H}
\end{array}\right] V^{\prime}=\left[\begin{array}{c}
0 \\
W_{H} V
\end{array}\right]
$$

where, $W_{L}$ and $W_{H}$ are the diagonal weighting matrices that balance the contraction and attraction constraints, respectively.

For the equation 2, a single solution does not make the mesh to the approximately $1 \mathrm{D}$ centerline and taking the following methods for iterative contraction ${ }^{[26]}$.

(1) Calculate the average area $A$ of the model triangle and the model volume $\mathrm{Vol}_{0}$.

(2) Let $W_{H, i}^{0}=1.0, W_{L, i}^{0}=\frac{1}{10 \sqrt{A}}$.

(3) Calculating the current Laplacian matrix $L^{t}$.

(4) Solve the equation $\left[\begin{array}{c}W_{L}^{t} L^{t} \\ W_{H}^{t}\end{array}\right] V^{t+1}=\left[\begin{array}{c}0 \\ W_{H}^{t} V^{t}\end{array}\right]$ for the new vertex $V^{t+1}$.

(5) Update $W_{H, i}^{t+1}=W_{H, i}^{0} \sqrt{S_{i}^{0} / S_{i}^{t}}, W_{L}^{t+1}=r W_{L}^{t}$, where $S_{i}^{0}$ and $S_{i}^{t}$ are the sums of the area of the triangle adjacent to the point $v_{i}$, and $r$ defaults to 2.0 .

(6) Calculate the current volume of the model $\mathrm{Vol}_{t}$.

(7) If $k=\frac{V l_{t}}{V o l_{0}}<10^{-5}$, the iteration is terminated, and the model after the contraction to output, otherwise it returns to step 3 .

Different root models are tested, the models can be iterated to within 10 times. Figure 6 is a set of root contraction processes. When iterating to the fifth time, the $k$ value is less than the threshold, and the iteration is terminated.

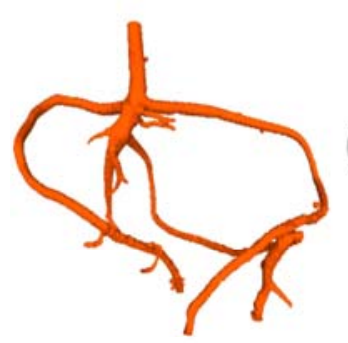

Input

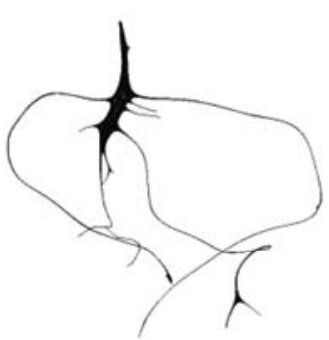

$1 \mathrm{st}$ $k=0.1045$

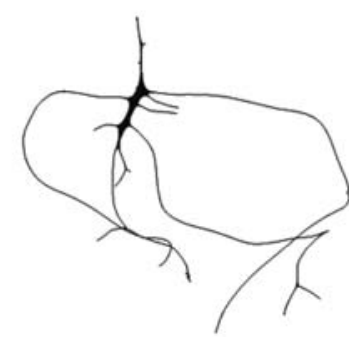

2nd

$k=0.0125$

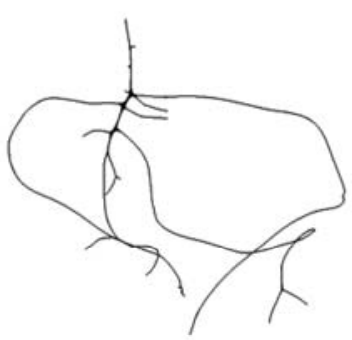

3 rd

$k=0.0003$

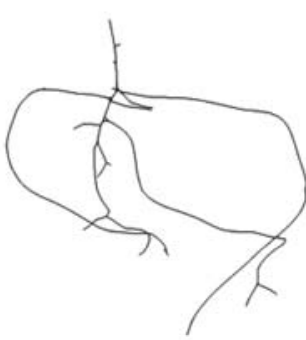

5 th

$k=5.54 \times 10^{-6}$

Figure 6 Process of mesh contraction

\subsubsection{Mesh removal}

For the graph obtained by contraction, its shape approximates a 1D curve. However, it contains all vertex and connection information, not a 1D centerline. So, a mesh removal method needs to take to acquire the $1 \mathrm{D}$ centerline. In the process of removing the mesh, to maintain the topological connection of the mesh, the half-edge collapse method is adopted for removal ${ }^{[26,35-36]}$.
After the mesh is removed, the vertexes for representing the 1D centerline and the connection relationship between the vertexes is obtained.

2.2.4 Post-processing for centerline

2.2.4.1 Branch construction

For the obtained centerline, its data structure is an undirected graph, and only contains the location information of the points and 
the connection information between the points, and there is no branch topology information. For the points, it contains three types of adjacency relation. As shown in Figure 7. The first one is the endpoints, it has only one neighbor. The second one is the common points, it has two neighbors. The third one is the branching points, it has at least three neighbors.

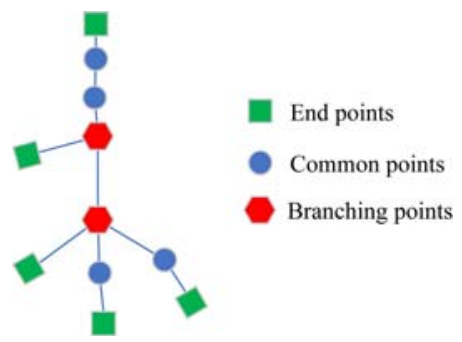

Figure 7 Three types of points for the centerline

Therefore, a branch construction method based on depth searches is proposed. First, iterating through the third type of points, and the deep search is started from each point in turn. The search path is saved to the queue until the non-second type of points is encountered. Then, finding the repeated path in the queue and removing it, and each of these paths can be considered as a branch. Third, looking for the branches with the same head or tail points in the queue, and merging the two branches with the angle smaller than the setting angle threshold. Finally, the average moving distance of the points in each branch is calculated, and the branch with the largest moving distance is taken as the main branch. Starting from the main branch, the parent-child branch relationships are constructed for the branches in the queue through the connection relationship between the head and tail points of different branches.

\subsubsection{Optimization of the centrality}

Due to the imbalance of the contraction and attraction constraints during the contraction process in local detail, it may acquire a centerline that is off center and even goes outside the mesh $^{[26]}$. For the endpoints, the main problem of it is moves to the inside of the mesh during the contraction process and resulting in the loss problem, as shown in Figure 8a. For the common points, the main problem is off center, as shown in Figure $8 \mathrm{~b}$. For the branching points, the main problem is to move to a smaller branch, as shown in Figure 8c.

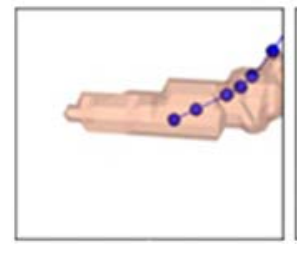

a.

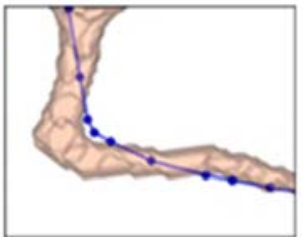

b.

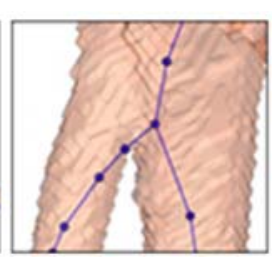

c.
Figure 8 Problems of the centerline

First, a global operation is implemented. And the method is moving each point to the $L_{1}$ medial point of its corresponding local mesh region ${ }^{[25]}$.

$$
v_{i}^{\prime}=\arg \min _{x} \sum_{j \in N(i)}\left\|x-v_{j}\right\|
$$

where, $v_{i}^{\prime}$ is the optimized coordinates of the $i$ point, and $N(i)$ is the adjacency list of the $i$ point, and $v_{j}$ is the coordinates of the $j$ point in the original mesh.

For the $L_{1}$ medial point, it is robust. However, its calculation is complicated. Therefore, in the case of having a good mesh quality, the method directly calculates the average position of the adjacent points. Where $n$ is the number of the current adjacency list.

$$
v_{i}^{\prime}=\frac{\sum_{j \in N(i)} v_{j}}{n}
$$

For the endpoints, its correct position is considered to be on the extension of the direction that combines with the adjacent point. Therefore, moving it to the position where the extension line meets the surface mesh. For the branching points, moving it to the centroid position of the adjacent point in its different branches.

In summary, after the optimization of centrality, a better quality centerline is obtained. Figure 9 shows the comparison before and after the optimization. The blue in the figure is the centerline before the optimization, and the red is optimized centerline.

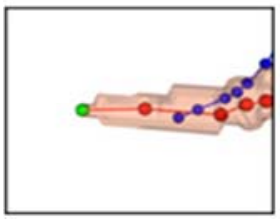

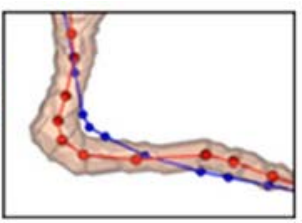

b.

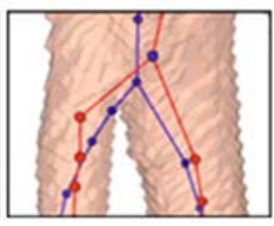

c.
Figure 9 Optimization of centrality for the centerline

\subsubsection{Centerline smoothing}

To increase the smoothness of the centerline, a Cubic B Spline fit is performed on it. For each individual branch, the centerline points are used as the control points, and a B-spline curve is generated by approaching a set of control points ${ }^{[37,38]}$.

To save the centerline and perform the architecture parameters calculation, the fitted spline curve is downsampled. By default, the sampling is performed at equal intervals by 3 times the number of original points, thereby obtaining a new point. The obtained new points are connected by the straight line. So that a centerline with a relatively smooth distance and substantially equal distance between the branch nodes can be obtained under the premise of less loss of precision.

\subsubsection{Calculation of root architecture parameter}

Common root 3D architecture parameters include root branch number, root branch length, root branch angle, root branch surface area, and volume. The length of the root branches and the angle of the root branches are the basic parameters of the root architecture. The length of branches and the angle of branches are calculated as follows.

For the $\mathrm{n}$ points $v_{i}(i=1 \ldots n)$ in a branch, the branch length is the sum of the distances between the points, and the branch length $L_{b}$ :

$$
L_{b}=\sum_{i=1}^{n-1}\left|\overrightarrow{v_{l} v_{l+1}}\right|
$$

For the angle of the branches, it is defined as the angle formed by the tangential direction at the common points of the two branches as the angle of the root branch. Therefore, the tangent vectors of the two branches are first calculated separately, and the angle of the root branch is calculated by the tangent vector. For the two branch tangent vectors $\gamma_{1}$ and $\gamma_{2}$, the angle $\theta$ :

$$
\theta=\arccos \left(\frac{\gamma_{1} \gamma_{2}}{\left|\gamma_{1} \| \gamma_{2}\right|}\right)
$$

\section{Experiments and results}

To validate the method proposed in this paper, two experiments were performed. Firstly, the centerline extraction results were visually and quantitatively assessed. Secondly, the root architecture parameters were calculated by the centerline and compared to the manual measurement results. In addition, the 
time consumption of the algorithm was calculated to test the real-time performance of this method.

The test equipment included the following: a laptop with 8GB RAM and Intel(R) Core(TM)i7-3632 QM CPU@2.20 GHz, a Windows 10 operating system, VTK 8.0, OpenCV 2.4.13, Qt 5.8.0, Visual Studio 2013 programming environment and the $\mathrm{C}++$ programming language.

\subsection{Centerline extraction experiment results}

Four sets of plant roots with different architecture were collected and transplanted to flower pots while maintaining their original conditions. And placing them in the XCT device for scanning, thereby obtaining four sets of root CT images. After that, the centerline was obtained by the above series of algorithms. The centerline extraction results were shown in Figure 10.

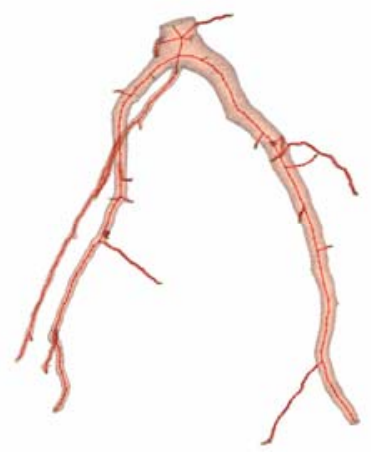

a.

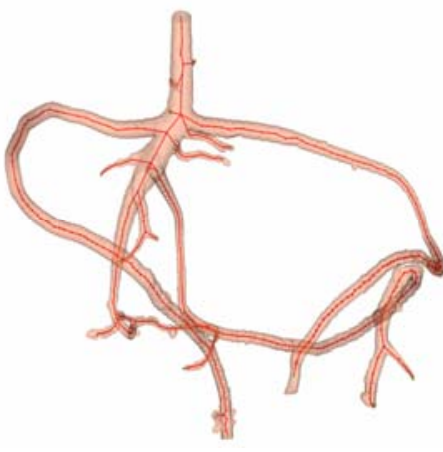

b.
Figure 10 Results of the centerline extraction

\subsection{Compare with manual measurements}

\subsubsection{Error measured}

In this section, the branch length and angle calculated by the system are compared with the experimental results of the model artificial mouse in the 3D software to characterize the system measurement accuracy. And calculating the errors at the same time, as well as the mean absolute percentage error (MAPE) and root mean square error (RMSE) of the measurement results.

$$
\begin{gathered}
\text { MAPE }=\frac{1}{n} \sum_{i=1}^{n} \frac{\left|x_{s i}-x_{m i}\right|}{x_{m i}} \times 100 \% \\
R M S E=\sqrt{\frac{1}{n} \sum_{i=1}^{n}\left(x_{s i}-x_{m i}\right)^{2}}
\end{gathered}
$$

where, $x_{s i}$ represents the system measurement result and $x_{m i}$

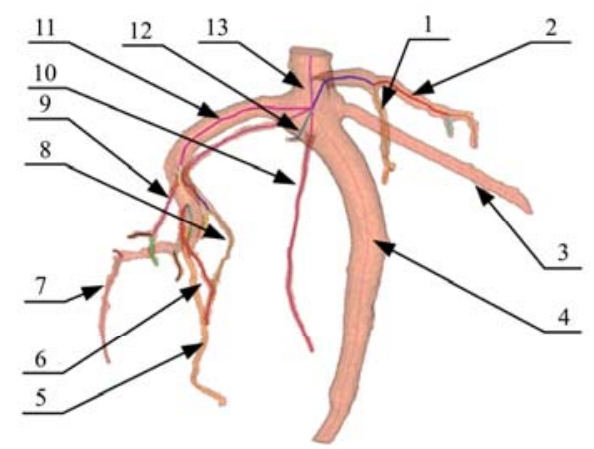

Figure 11 Length measurement of root's branches

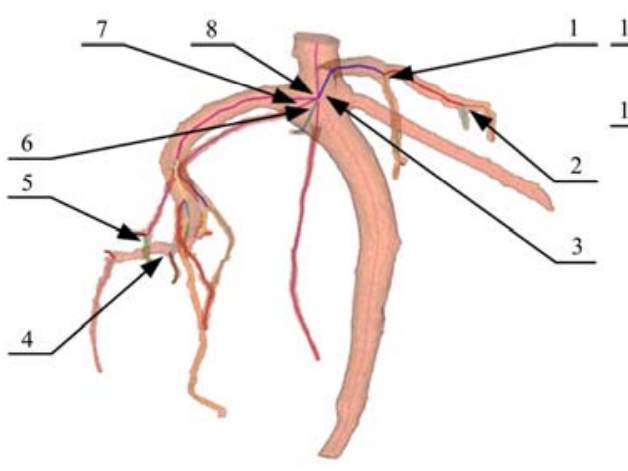

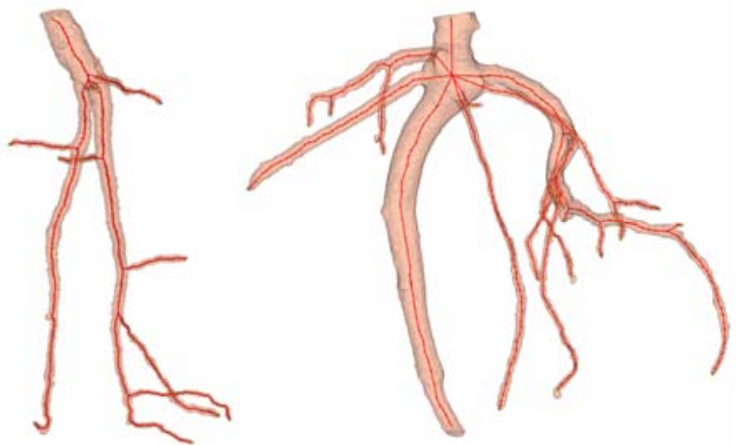

d. represents the manual measurement result.

\subsubsection{Experiment results}

According to the method of Section 2.2.5, the length and angle of the root branch automatically calculated by the algorithm were compared with the manual measurement results. Therefore, a typical root was chosen to illustrate the accuracy of the method. The length measurement of the root branch marked as shown in Figure 11a was carried out, and the measurement results were shown in Figure 11b. The MAPE of the branch length was $1.74 \%$, and the RMSE was 0.006. The angle of the root branch marked as shown in Figure 12a was measured, and the measurement result was shown in Figure 12b. The MAPE of the branch angle was $4.51 \%$ and the RMSE was 4.095 .

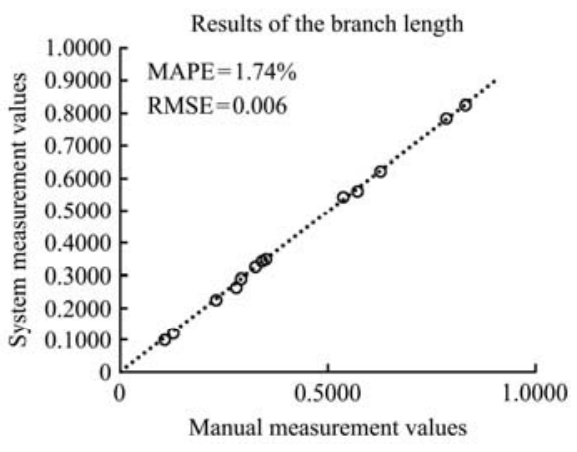

b.

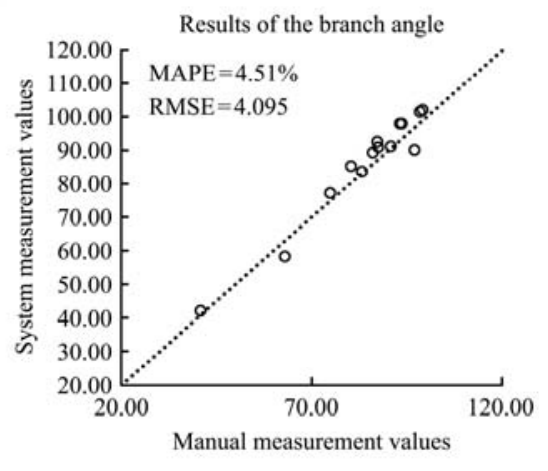

b.

Figure 12 Angle measurement of root's branches 


\section{Discussion}

For the centerline extraction, the method does not require a cumbersome parameter adjustment process. Through the pre-processing and post-processing steps, the centerline maintains a good centrality and branches connection, and a higher quality of centerline is obtained. However, due to the image segmentation problem, some noise still exists on the surface of the pre-processed model, so that some noise points are preserved during the contraction process, resulting in redundant branches. Therefore, for the noise problem, further work can be handled from the following two aspects. On the one hand, the segmentation quality of the root CT slice images needs to be further improved. On the other hand, a smoothing method of the pre-processing method needs to be added.

For the experiment, the comparison between the manual measurement shows the accuracy of the calculation of the root architecture parameters in this paper. The causes of error include the accuracy of centerline extraction and the accuracy of manual measurement.

For the branch angle, the most important factor is the offset of the centerline's points, and the smaller offset of the points can cause a large error with the actual value. At the same time, the result of manual measurement will also cause an error between the actual values. For reducing the error, it is necessary to further improve the accuracy of the centerline extraction to reduce the influence of the points offset problem. And performing multiple measurements to calculate the average value to reduce the error of manual measurement.

For the branch length, the main cause of the error is the loss problem of the end points. Although an extension algorithm was adopted to solve this problem, in a sense, the extension does not result in a true endpoint position, which also causes an error between the actual values.

For the whole algorithm, the 3D images preprocessing and reconstruction are cumbersome, and which requires a lot of computing resources. For the centerline extraction part, the solution of the contraction matrix equation from multiple times is the step that takes the most amount of time. The time complexity for the decomposition of $L D L^{T}$ is calculated as $O\left(V^{3}\right)$, where $V$ is the number of vertices of the input mesh. For the post-processing, the processing object is the obtained centerline' points, which the number of it is far less than the number of the grid points, edges and faces, so it can be ignored in the calculation.

\section{Conclusions}

In order to obtain the $3 \mathrm{D}$ architecture of plant roots, this paper studied a root architecture measurement method based on XCT and centerline extraction method. The acquired root $\mathrm{CT}$ images were segregated-firstly. The 3D surface mesh model was reconstructed after a pre-processing process. On this basis, the root centerline was obtained by mesh contraction, and the centerline with branch topology was obtained by post-processing steps. Four groups of different architecture of roots were tested, and the results of root architecture calculations were compared with manual measurements. The results show that the method can obtain accurate root architecture parameters. It provides an effective method for the detection of plant root architecture, which has important practical significance for the study of plant roots and the improvement of crop yields.

\section{Acknowledgments}

This work was supported by a grant from the National Natural Science Foundation of Guangdong Province, China (No. 2018B030306026) and National Natural Science Foundation of China (No. 31271609).

\section{[References]}

[1] Zhou X, Luo X. 3D Visualization of Root System in Situ Based on XCT Technology. Transactions of the Chinese Society for Agricultural Machinery, 2009; 40, 202-205. (in Chinese with English abstract)

[2] Goss M J, Miller M H, Bailey L D, et al. Root growth and distribution in relation to nutrient availability and uptake. European Journal of Agronomy, 1993, 2(2), 57-67. doi: 10.1016/S1161-0301(14)80135-4.

[3] Watt M, Wasson A, Chochois V, et al. Root-based solutions to increasing crop productivity, CRC Press, 2013; 21.1-21.17.

[4] Zhou W, Li C, et al.. Research Method of Crop Phenotypic, Science China Press, 2017; 86-130.

[5] Böhm W. Methods of studying root systems. Springer Science \& Business Media, 1979. doi: 10.1007/978-3-642-67282-8.

[6] Roose T, Keyes S D, Daly K R, et al. Challenges in imaging and predictive modeling of rhizosphere processes, Plant and Soil, 2016; 407(1-2): 9-38. doi: 10.1007/s11104-016-2872-7.

[7] French A, Ubedatomas S, Holman $\mathrm{T} J$, et al. High-Throughput Quantification of Root Growth Using a Novel Image-Analysis Tool. Plant Physiology, 2009; 150(4): 1784-1795. doi: 10.1104/pp.109.140558.

[8] Armengaud P, Zambaux K, Hills A, et al. EZ-RHIZO:integrated software for the fast and accurate measurement of root system architecture. The Plant Journal, 2009; 57(5): 945-956. doi: 10.1111/j.1365-313X.2008.03739.x.

[9] Lobet G, Pages L, Draye X. A Novel Image-Analysis Toolbox Enabling Quantitative Analysis of Root System Architecture. Plant Physiology, 2011; 157(1): 29-39. doi: 10.1104/pp.111.179895.

[10] Pierret A, Gonkhamdee S, Jourdan C, et al. IJ_Rhizo:an open-source software to measure scanned images of root samples. Plant and Soil, 2013; 373(2): 531-539. doi: 10.1007/s11104-013-1795-9.

[11] Das A, Schneider H, Burridge J, et al. Digital imaging of root traits (DIRT): a high-throughput computing and collaboration platform for field-based root phenomics. Plant methods, 2015; 11(1): 51 . doi: 10.1186/s13007-015-0093-3.

[12] Wasson A, Bischof L, Zwart A, et al A portable fluorescence spectroscopy imaging system for automated root phenotyping in soil cores in the field. Journal of Experimental Botany, 2016; 67(4): 1033-1043. doi: $10.1093 /$-jxb/erv570.

[13] $\mathrm{Xu} \mathrm{Z,} \mathrm{Valdes} \mathrm{C,} \mathrm{Clarke} \mathrm{J.} \mathrm{Existing} \mathrm{and} \mathrm{Potential} \mathrm{Statistical} \mathrm{and}$ Computational Approaches for the Analysis of 3D CT Images of Plant Roots. Agronomy, 2018; 8(5): 71. doi: 10.3390/agronomy8050071.

[14] Gregory P J, Hutchison D J, Read D B, et al. Non-invasive imaging of roots with high resolution X-ray micro-tomography. Plant and Soil, 2003; 255(1): 351-359. doi: 10.1023/a:1026179919689.

[15] Bingham I J, Wu L. Simulation of wheat growth using the $3 \mathrm{D}$ root architecture model SPACSYS: Validation and sensitivity analysis, European Journal of Agronomy, 2011; 34(3): 181-189. doi: 10.1016/j.eja.2011.01.003.

[16] Dunbabin V M, Postma J A, Schnepf A, et al. Modelling root-soil interactions using three-dimensional models of root growth, architecture and function. Plant and Soil, 2013; 372(1-2): 93-124. doi: 10.1007/s11104-013-1769-y.

[17] Wu J, Pagès L, Wu Q, et al., 2015. Three-dimensional architecture of axile roots of field-grown maize. Plant and Soil, 387(1-2), 363-377. doi: 10.1007/s11104-014-2307-2.

[18] Wu Q, Wu J, Zheng B, et al.. Optimizing soil-coring strategies to quantify root-length-density distribution in field-grown maize: virtual coring trials using 3-D root architecture models. Annals of Botany, 2017; 121(5): 809-819. doi: 10.1093/aob/mcx117.

[19] Koebernick N, Weller U, Huber K, et al.. In situ visualization and quantification of three-dimensional root system architecture and growth using X-Ray computed tomography. Vadose Zone Journal, 2014; 3(24): 13(8). doi: 10.2136/vzj2014.03.0024.

[20] Metzner R, Eggert A, Van Dusschoten D, et al. Direct comparison of MRI and X-ray CT technologies for 3D imaging of root systems in soil: potential and challenges for root trait quantification. Plant methods, 2015; 11(1): 17 . doi: 10.1186/s13007-015-0060-z. 
[21] Landl M, Schnepf A, Vanderborght J, et al. Measuring root system traits of wheat in 2D images to parameterize 3D root architecture models. Plant and Soil, 2018; 425: 457-477. doi: 10.1007/s11104-018-3595-8.

[22] Van Dael M, Verboven P, Dhaene J, et al. Multisensor X-ray Inspection of Internal Defects in Horticultural Products. Postharvest Biology and Technology, 2017; 128, 33-43. doi: 10.1016/j.postharvbio.2017.02.002.

[23] Pfeifer J, Kirchgessner N, Colombi T, Walter A. Rapid phenotyping of crop root systems in undisturbed field soils using X-ray computed tomography. Plant Methods, 2015; 11(1): 41. doi: 10.1186/ s13007-015-0084-4.

[24] Grélard F, Baldacci F, Vialard A, et al. Centerlines of Tubular Volumes Based on Orthogonal Plane Estimation. International Conference on Discrete Geometry for Computer Imagery, 2016; 427-438. doi: 10.1007/978-3-319-32360-2 33.

[25] Huang H, Wu S, Cohen-Or D, et al. L1-Medial Skeleton of Point Cloud. Acm Transactions on Graphics, 2013; 32(4), 65. doi: 10.1145/ 2461912.2461913.

[26] Au K C, Tai C L, Chu H K, et al. Skeleton Extraction by Mesh Contraction. Acm Transactions on Graphics, 2008; 27(3), 44. doi: 10.1145/1360612.1360643.

[27] Lee T C, Kashyap R L, Chu C N. Building Skeleton Models via 3-D Medial Surface Axis Thinning Algorithms. CVGIP: Graphical Models and Image Processing, 1994; 56(6): 462-478. doi: 10.1006/ cgip.1994.1042.

[28] Ginat D T, Gupta R. Advances in Computed Tomography Imaging Technology. Annual Review of Biomedical Engineering, 2014; 16(16): 431. doi: 10.1146/annurev-bioeng-121813-113601.

[29] Lafond J A, Liwen H, Pierre D. Concepts and analyses in the CT scanning of root systems and leaf canopies: A timely summary. Frontiers in Plant Science, 2015; 6, 1111. doi: 10.3389/fpls.2015.01111.

[30] Sharma N, Aggarwal L. Automated medical image segmentation techniques. Journal of Medical Physics, 2010; 35, 3-14. doi: 10.4103/ 0971-6203.58777.

[31] Diels E, Van Dael M, Keresztes J, et al. Assessment of bruise volumes in apples using X-ray computed tomography. Postharvest Biology and Technology, 2017; 128, 24-32. doi: 10.1016/j.postharvbio.2017.01.013.

[32] Van Dael M, Lebotsa S, Herremans E, et al. A segmentation and classification algorithm for online detection of internal disorders in citrus using X-ray radiographs. Postharvest Biology and Technology, 2016; 112, 205-214. doi: 10.1016/j.postharvbio.2015.09.020

[33] Zhou Z, Zhou X, Luo X, et al. Study on Matlab-based Segmentation Algorithms for X-ray CT Image of Plant Root. Transactions of the Chinese Society for Agricultural Engineering, 2006; 37(12): 118-121 (in Chinese with English abstract).

[34] Sorkine O. Laplacian mesh processing, Eurographi. 2005; 53-70.

[35] Tagliasacchi A, Alhashim I, Olson M, et al. Mean Curvature Skeletons. Computer Graphics Forum, 2012; 31(5): 1735-1744. doi: 10.1111/ j.1467-8659.2012.03178.x.

[36] Botsch M, Kobbelt L, Pauly M, et al. Polygon Mesh Processing. CRC press, 2010.

[37] Hearn D, Baker M P. Computer Graphics with OpenGL. Publishing House of Electronics Industry, 2012; 91-120.

[38] Garland M, Heckbert P S. Surface simplification using quadric error metrics. Proceedings of the 24th annual conference on Computer graphics and interactive techniques, 1997; 209-216. doi: 10.1145/ 258734.258849. 